Cite this: Chem. Sci., 2013, 4, 2760

Received 1st March 2013

Accepted 9th April 2013

DOI: $10.1039 / c 3 s c 50587 a$

www.rsc.org/chemicalscience

\title{
Discovery of an iridacycle catalyst with improved reactivity and enantioselectivity in the hydrogenation of dialkyl ketiminest
}

\author{
York Schramm, Fabiola Barrios-Landeros $\ddagger$ and Andreas Pfaltz* \\ Catalytically active iridacycles are formed by cyclometalation of acetophenone imines with Ir-PHOX \\ complexes under hydrogen atmosphere. These complexes show unusually high reactivity and \\ enantioselectivity in the hydrogenation of alkyl methyl ketimines. The structure of the cyclometalated \\ imine has a strong effect on the conversion and enantiomeric excess.
}

\section{Introduction}

Chiral amines play an important role as building blocks for the synthesis of pharmaceuticals and agrochemicals. They are also of great importance as chiral auxiliaries, catalysts and resolving agents. Therefore, asymmetric hydrogenation of ketimines has received much attention as an attractive, very direct route to enantiomerically enriched amines. ${ }^{1}$ High yields, perfect atom economy and mild conditions make this approach ideal for industrial applications. This is impressively demonstrated by the multi-ton scale production of the herbicide metolachlor, based on an extremely active and productive Ir-diphosphine catalyst. $^{2}$

During the last two decades a wide range of chiral $\mathrm{Ti}, \mathrm{Rh}, \mathrm{Ir}$, $\mathrm{Pd}, \mathrm{Ru},{ }^{1}$ and most recently $\mathrm{Fe}^{\mathbf{1 , 3}}$ complexes have been developed that catalyze the hydrogenation of various imines with high enantioselectivity. However, the scope of most catalysts is rather narrow and there are still important classes of imines that give unsatisfactory results with the available catalysts. Especially the hydrogenation of imines derived from dialkyl ketones remains a challenging problem. With the exception of the dual catalyst system reported by Xiao and co-workers, ${ }^{4}$ consisting of a chiral $\operatorname{Ir}\left(\mathrm{Cp}^{*}\right)$-diamine complex and an elaborate chiral binaphtholderived phosphoric acid (TRIP), most catalysts give very low enantioselectivities with these substrates. Organocatalytic asymmetric imine reduction and reductive amination has also been developed to afford chiral aliphatic amines with high enantioselectivities. ${ }^{5}$ However, these reactions generally suffer

University of Basel, Department of Organic Chemistry, St. Johanns-Ring 19, 4056 Basel, Switzerland. E-mail: andreas.pfaltz@unibas.ch; Fax: +41 61 2671103; Tel: $+41612671108$

$†$ Electronic supplementary information (ESI) available: Protocols for procedures and experimental data. CCDC 916564. For ESI and crystallographic data in CIF or other electronic format see DOI: 10.1039/c3sc50587a

$\ddagger$ Current address: Yeshiva University, Department of Chemistry, New York, NY, 10033, USA. from lower yields and very long reaction times compared to transition-metal catalyzed hydrogenations. Furthermore, they require hydride donors such as dihydropyridines that generate stoichiometric waste products. So a practical readily accessible catalyst for the asymmetric hydrogenation of dialkyl ketimines remains elusive.

We have recently begun to reinvestigate Ir-phosphinooxazoline complexes that we originally introduced as catalysts for imine hydrogenation in 1997. ${ }^{6 \boldsymbol{a}}$ After evaluation of a wide range of phosphinooxazoline (PHOX) derivatives and careful optimization of the reaction conditions, excellent enantioselectivities and high turnover numbers have been achieved in the hydrogenation of aryl alkyl $N$-arylketimines such as I1 (Scheme 1) ${ }^{6 \boldsymbol{b}}$

However, analogous dialkyl ketimines still gave disappointing results. We therefore decided to conduct a mechanistic study, in the hope that it would guide the development of improved catalysts with broader substrate scope.

\section{Results and discussion}

Here we report the outcome of this study that led to surprising insights into the structure of the catalytic intermediates and, ultimately, to a new catalyst system that gave promising results in the hydrogenation of dialkyl ketimines.

Experimental mechanistic studies of imine hydrogenation with Ir-PHOX complexes have not been reported. However,

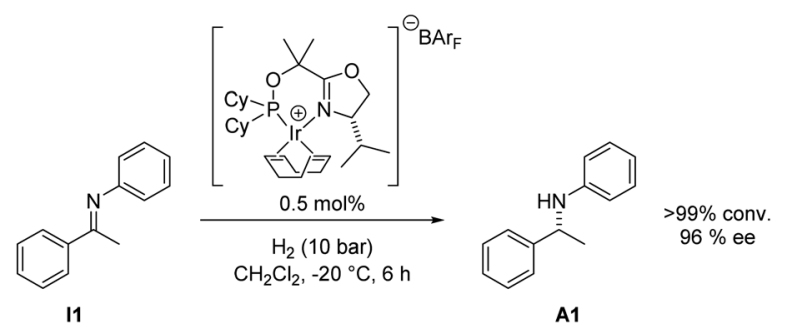

Scheme 1 Asymmetric hydrogenation of acetophenone N-phenylimine I1. 
Hopmann and Bayer ${ }^{7}$ carried out DFT calculations with catalyst 1a on nine different catalytic cycles based on proposed mechanisms for imine hydrogenations with other types of Ir complexes. The computed energies of the turnover-limiting transition states indicated a pathway involving proton transfer from an Ir-hydride complex to a free, uncoordinated imine, followed by hydride transfer to the resulting iminium ion. As all proposed mechanisms included Ir-dihydride complexes as intermediates, we decided to prepare a dihydride complex from the $\operatorname{Ir}(\mathrm{PHOX})$ precursor 1a following the procedure of Mazet et $a .^{8}$ and to study its reactivity with $\mathbf{I 1}$ as a typical substrate (Scheme 2).

When the dihydride complex, generated by reaction of 1a with $\mathrm{H}_{2}$ in THF at $-25^{\circ} \mathrm{C}$, was treated with $\mathbf{I} 1$ at $0{ }^{\circ} \mathrm{C}$, rapid formation of an iridacycle 2 was observed. This complex proved to be very sensitive and rapidly decomposed upon exposure to air. Attempts to obtain suitable crystals for X-ray analysis failed and, therefore, the BAr $_{F}$ (tetrakis[(3,5-trifluoromethyl)phenyl]borate) counterion that, in our experience, often impedes crystallization, was exchanged with chloride or hexafluorophosphate. The chloride complex 3 was readily obtained by treatment with $\mathrm{LiCl}$ and silica gel in ethyl acetate and purified by flash chromatography on silica gel. Ion exchange with $\mathrm{NaBAr}_{\mathrm{F}}$ in THF led back to the $\mathrm{BAr}_{\mathrm{F}}$ complex 2.

While crystallization attempts of 3 were unsuccessful, the hexafluorophosphate salt 5 furnished suitable crystals for X-ray analysis (Scheme 3 and Fig. 1). ${ }^{9}$ Furthermore, a preliminary crystal structure of the analogous iridacycle 7 prepared from SimplePHOX complex 6 (Scheme 4 and Fig. 2) could also be determined. $^{\mathbf{1 0}}$

An analogous cyclometalation reaction of $\left[\operatorname{Ir}(\mathrm{H})_{2}\left(\mathrm{PPh}_{3}\right)_{2^{-}}\right.$ (acetone) $\left.{ }_{2}\right] \mathrm{PF}_{6}$ with benzaldehyde $\mathrm{N}$-benzylimine has been reported by James and co-workers. ${ }^{11}$ The resulting iridacycle was tested in the hydrogenation of imines but showed no catalytic activity. ${ }^{11 a}$

When complex 3 was tested as catalyst for the hydrogenation of $\mathbf{I 1}$, no reaction was observed. However, when the chloride was replaced with the non-coordinating anion $\mathrm{BAr}_{\mathrm{F}}$ by addition of an equimolar amount of $\mathrm{NaBAr}_{\mathrm{F}}$, an active catalyst was generated that furnished the same enantiomer of amine A1 with identical enantioselectivity as the reduction catalyzed by the parent complex 1a (Scheme 5).

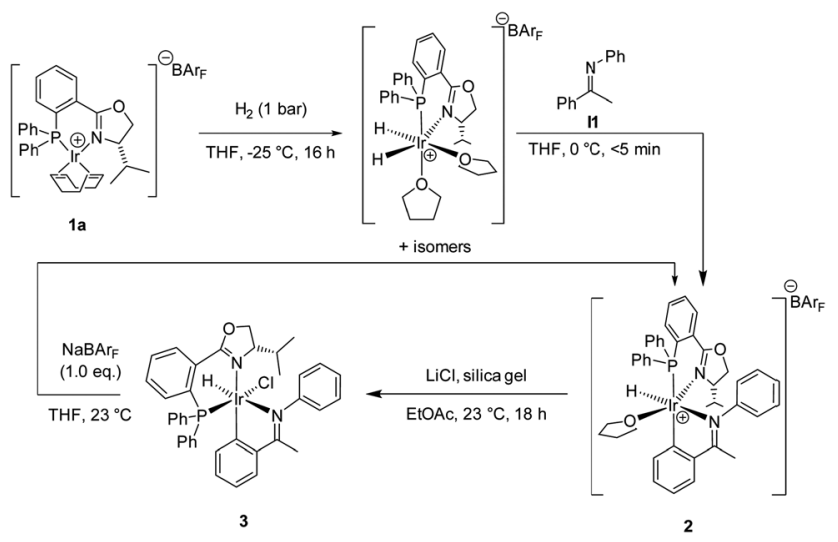

Scheme 2 Formation of cyclometalated imine complexes.

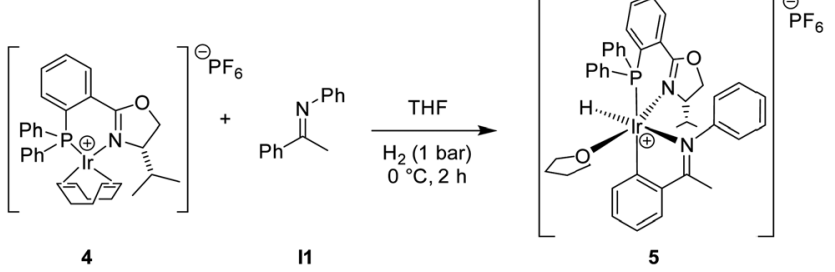

Scheme 3 Preparation of iridacycle $\mathbf{5}$

What are the possible conclusions that can be drawn from these results? The iridacycle formed under hydrogenation conditions could be an inactive species outside the catalytic cycle that is in equilibrium with an active catalytic intermediate through reversible cyclometalation/reductive elimination, similar to the reaction scheme proposed by Marcazzan and James. $^{12}$

Alternatively, it could be directly involved in the catalytic cycle. In this case, it could either react via reduction of the cyclometalated imine, followed by reductive elimination releasing the saturated amine, or the cyclometalated imine could serve as a stable ligand that remains bound throughout the catalytic cycle.

To distinguish between these possibilities we carried out the cross-over experiment shown in Scheme 6. If a catalytic intermediate derived from $\mathbf{3}$ would release the free imine I1, the cyclometalation product of substrate $\mathbf{2} 2$ along with amine A1 would be formed. However, we did not observe even traces of $\mathbf{I 1}$ or $\mathbf{A 1}$ in the course of the hydrogenation reaction by GC analysis. $^{\mathbf{1 3 , 1 4}}$ These results are consistent with the hypothesis that cyclometalation is irreversible and the imine remains bound to iridium throughout the catalytic reaction. The enantiomeric excess of $\mathbf{A} 2$ remained constant throughout the reaction. ${ }^{13}$

We speculated that the cyclometalated complex formed under hydrogenation conditions could be a superior catalyst compared to complex $\mathbf{1 a}$ and that the poor results in the hydrogenation of aliphatic ketimines could be a consequence of the inability of these substrates to form cyclometalated complexes.

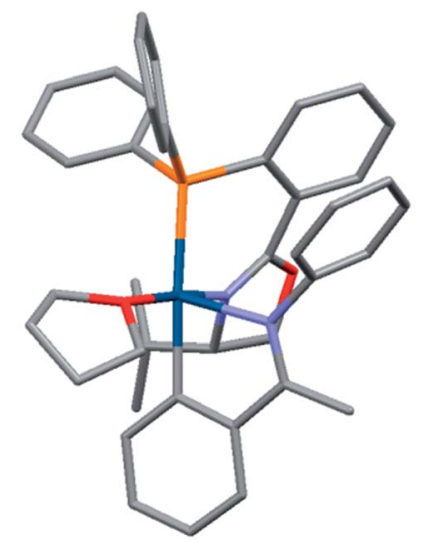

Fig. 1 Crystal structure of $\mathbf{5}$ ( $\mathrm{PF}_{6}$ counterion omitted for clarity). 


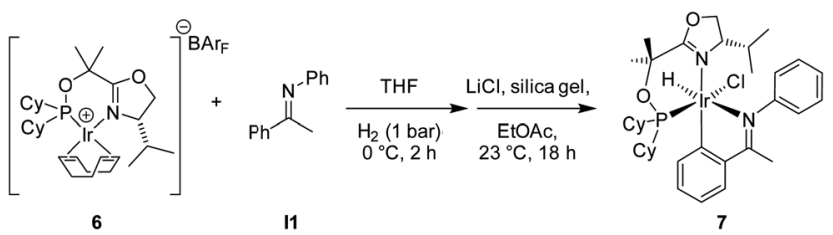

Scheme 4 Preparation of iridacycle $\mathbf{7}$.

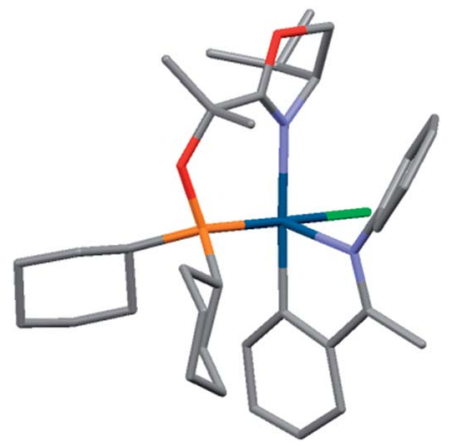

Fig. 2 Preliminary crystal structure of $\mathbf{7}$

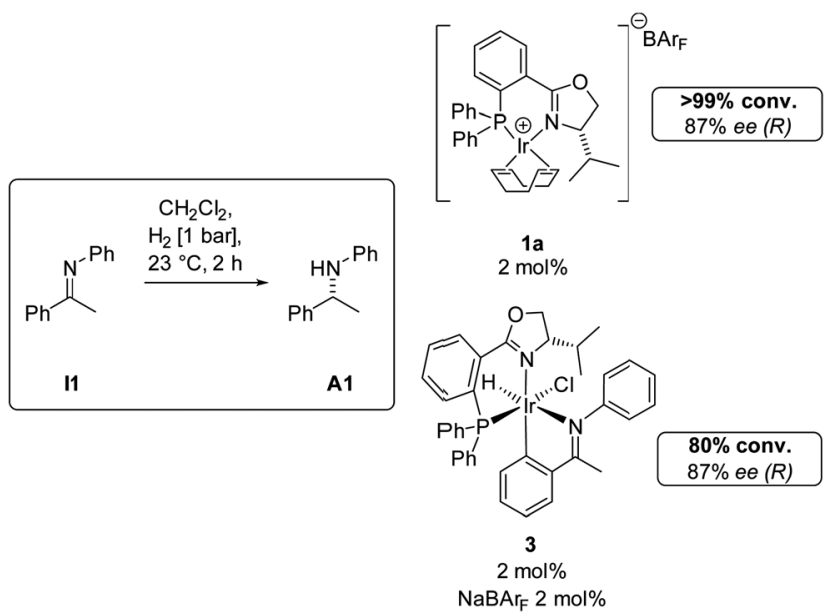

Scheme $\mathbf{5}$ Comparison of complexes $\mathbf{1 a}$ and $\mathbf{3}$ as precatalysts for the hydrogenation of imine $\mathbf{I 1}$.

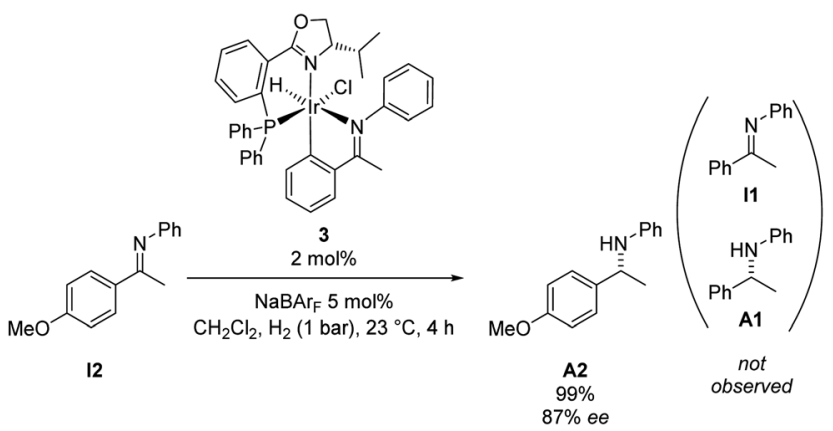

Scheme 6 Hydrogenation of imine $\mathbf{1 2}$ with iridacycle $\mathbf{3}$ in combination with NaBAr $r_{\text {. }}$
Indeed, the catalyst generated in situ from the iridacycle 3 by treatment with $\operatorname{NaBAr}_{\mathrm{F}}$ gave much higher conversion and enantioselectivity (Table 1, entry 1) in the hydrogenation of cyclohexyl methyl ketimine $\mathbf{3}$ than the parent Ir-PHOX complex 1a (entry 2) or iridacycle 3 alone (entry 3). Identical ee and higher conversion was obtained when the catalyst was generated by treating complex 1a with $\mathrm{H}_{2}$ and an equimolar amount

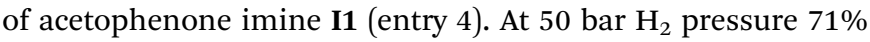
ee and full conversion were observed after activation with imine I1 (entry 5), compared to $27 \%$ conversion and $35 \%$ ee with complex 1a alone (entry 6).

These findings clearly indicate that the new more efficient catalyst is a cationic cyclometalated complex that arises by chloride abstraction from 3 or by reaction of precatalyst 1a with $\mathrm{H}_{2}$ and imine I1 ( $c f$. structure 2 in Scheme 2). Because cationic complexes such as $\mathbf{2}$ or $\mathbf{5}$ with very weakly coordinating anions proved to be too unstable and impractical to be used as catalysts, in situ activation of precatalyst $\mathbf{1 a}$ with acetophenone imine I1 or derivatives thereof was the method of choice for further experiments.

If the imine remains bound to the catalyst throughout the catalytic cycle, it is also involved in the enantiodiscriminating step. Cyclometalation of a chiral additive to an achiral complex would thus provide a chiral imine hydrogenation catalyst. We therefore prepared chiral complex $(S)$-11 derived from an achiral iridium-PHOX complex 8 and a chiral imine $\mathbf{9}$ (Scheme 7).

NMR analysis indicated the presence of two diastereomers of complex $(S)$-10. However, upon treatment with an equimolar amount of $\mathrm{NaBAr}_{\mathrm{F}}$ in $\mathrm{CD}_{2} \mathrm{Cl}_{2}$, a single hydride species $(S)$-11 was observed.

Both enantiomers of complex 11, formed in situ from the diastereomeric mixture of $(S)$-10ab or $(R)-\mathbf{1 0 a b}$, were tested as catalysts for the hydrogenation of I1 (Table 2). While low conversion was observed at atmospheric hydrogen pressure, hydrogenation at 5 bar afforded A1 with 54\% conversion (entry 3).

The resulting ee values showed a weak but reproducible influence of the chiral imine 9. Analogous catalysts derived from 1,3-benzoxazines were tested as well (Table 3) and gave reproducible enantioselectivities of up to $23 \%$ ee.

Table $\mathbf{1}$ Hydrogenation of imine $\mathbf{1 3}$ using catalyst $\mathbf{1} \mathbf{a}$ in the presence or absence of imine $\mathbf{I} \mathbf{1}$ or using iridacycle $\mathbf{3}$ in the presence or absence of $\mathrm{NaBAr}_{\mathrm{F}}$

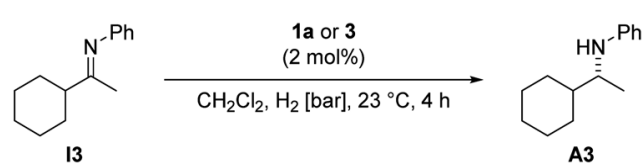

\begin{tabular}{lllccl} 
Entry & Catalyst & Additive & $p\left(\mathrm{H}_{2}\right) / \mathrm{bar}$ & Conv. $^{a}(\%)$ & $\mathrm{ee}^{b}(\%)$ \\
\hline 1 & $\mathbf{3}$ & NaBAr $_{\mathrm{F}}$ & 1 & 40 & $73(R)$ \\
2 & $\mathbf{1 a}$ & - & 1 & 5 & $69(R)$ \\
3 & $\mathbf{3}$ & - & 1 & 0 & - \\
4 & $\mathbf{1 a}$ & $\mathbf{I 1}(2 \mathrm{~mol} \%)$ & 1 & 50 & $73(R)$ \\
5 & $\mathbf{1 a}$ & $\mathbf{I 1}(2 \mathrm{~mol} \%)$ & 50 & $>99$ & $71(R)$ \\
6 & $\mathbf{1 a}$ & - & 50 & 27 & $35(R)$
\end{tabular}

${ }^{a}$ Determined by GC analysis. ${ }^{b}$ Determined by HPLC analysis on a chiral stationary phase. 


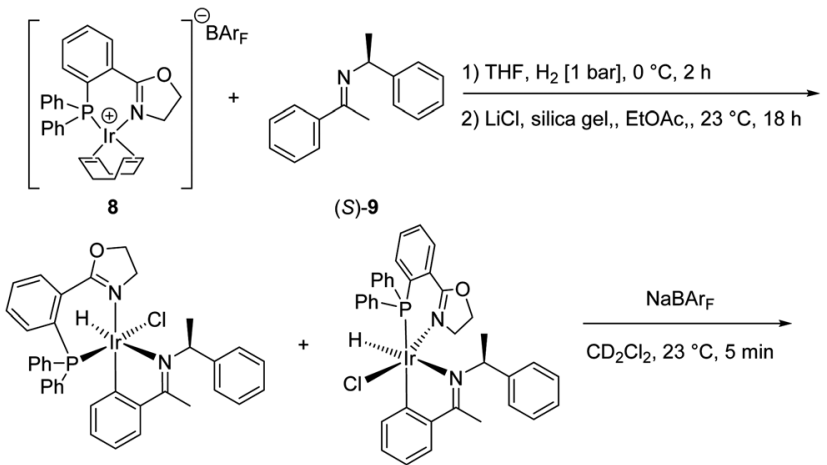

(S)-10a

(S)-10b

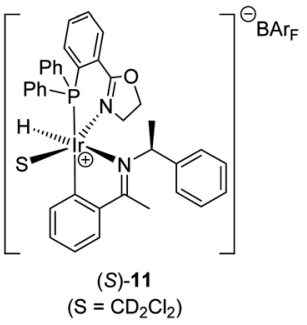

Scheme 7 Preparation of chiral iridium complexes (S)-10 derived from achiral IrPHOX complex 8 and chiral imine $\mathbf{9}$. Upon addition of $\mathrm{NaBAr}_{\mathrm{F}}$, chloride abstraction results in the formation of one single hydride complex (S)-11 in solution.

Table 2 Hydrogenation of imine $\mathbf{I} \mathbf{1}$ using iridacycle $\mathbf{1 0}$

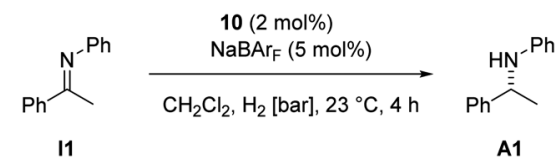

\begin{tabular}{lllll}
\hline Entry & Catalyst & $\mathrm{H}_{2} /$ bar & Conv. $^{a}(\%)$ & ee $^{b}(\%)$ \\
\hline 1 & $(S)-\mathbf{1 0}$ & 1 & 16 & $4(S)$ \\
2 & $(R)-\mathbf{1 0}$ & 1 & 18 & $6(R)$ \\
3 & $(S)-\mathbf{1 0}$ & 5 & 54 & $4(S)$ \\
4 & $(R)-\mathbf{1 0}$ & 5 & 44 & $6(R)$
\end{tabular}

${ }^{a}$ Determined by GC analysis. ${ }^{b}$ Determined by HPLC analysis on a chiral stationary phase.

Erosion of enantioselectivity was observed when hydrogenations were conducted at 5 bar (entries 2 vs. 3 and 4 vs. 5). Furthermore, complex 14 gave higher conversion but lower enantioselectivity than complex 13 (entries 2, 4 and 6). To get a more accurate correlation of the ee with conversion, the reaction was followed by GC and HPLC analysis (Fig. 3).

While complex 13 reacted with almost constant enantioselectivity, 14 showed a strong erosion of the enantioselectivity with increasing conversion. Furthermore, an overall higher conversion and faster reaction was observed for $\mathbf{1 4}$.

The following conclusions were drawn from these observations: while in 13 the benzoxazine remains bound to the iridium center throughout the reaction, complex $\mathbf{1 4}$ is not stable under the reaction conditions. As a consequence the benzoxazine
Table 3 Hydrogenation of imine I1 using iridacycle 12-14

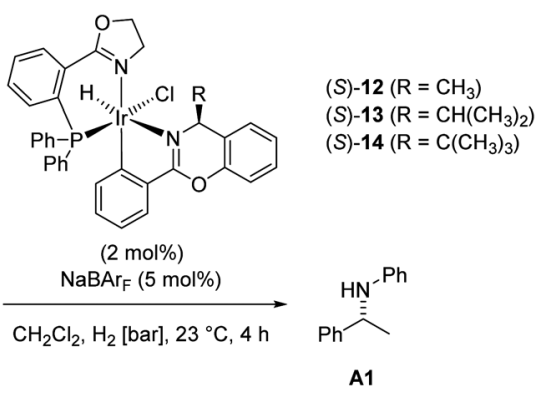

\begin{tabular}{lllll}
\hline Entry & Catalyst & $\mathrm{H}_{2} /$ bar & Conv. $^{a}(\%)$ & ee $^{b}(\%)$ \\
\hline 1 & $(S)-\mathbf{1 2}$ & 1 & 63 & $15(S)$ \\
2 & $(S)-\mathbf{1 3}$ & 1 & 31 & $23(R)$ \\
3 & $(S)-\mathbf{1 3}$ & 5 & 97 & $14(R)$ \\
4 & $(R)-\mathbf{1 3}$ & 1 & 20 & $23(S)$ \\
5 & $(R)-\mathbf{1 3}$ & 5 & 99 & $14(S)$ \\
6 & $(R)-\mathbf{1 4}$ & 1 & 40 & $17(S)$
\end{tabular}

${ }^{a}$ Determined by GC analysis. ${ }^{b}$ Determined by HPLC analysis on a chiral stationary phase.

ligand is replaced by imine $\mathbf{I} 1$ resulting in a complex with two achiral ligands, which produces racemic product. However, the high initial enantioselectivity of $44 \%$ ee clearly demonstrates that the cyclometalated ligand is involved in the enantiodiscriminating step of the reaction.

Attempts to improve the enantioselectivity by using catalysts prepared from a combination of chiral PHOX complexes with chiral benzoxazines gave disappointing results. However, systematic evaluation of various achiral $N$-aryl acetophenone imines as additives was more successful (Table 4).

The influence of substituents in the acetophenone phenyl ring showed no apparent trends that could be correlated with steric or electronic effects. Surprisingly, meta-substituents distinctly lowered the enantioselectivities (entries $4-8),{ }^{15}$ while an ortho-methyl or ortho-fluoro substituent had essentially no effect (entries 2 and 3). Overall, introduction of substituents in the acetophenone phenyl ring did not improve the enantioselectivity. In contrast, ortho-alkyl groups in the $N$-aryl ring resulted in enhanced

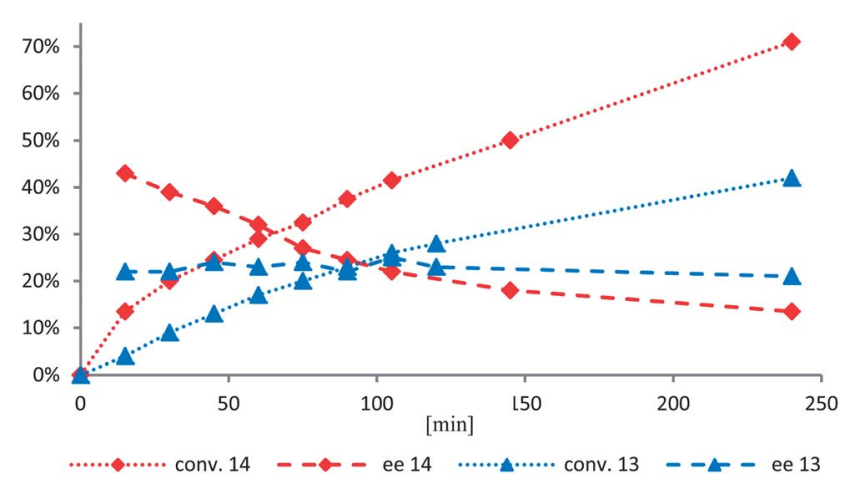

Fig. 3 Conversion (dotted) and enantioselectivity (dashed) of A1 using iridacycle 13 (blue triangles) and $\mathbf{1 4}$ (red diamonds) as the catalyst. 
Table 4 Screening of different imines as additives

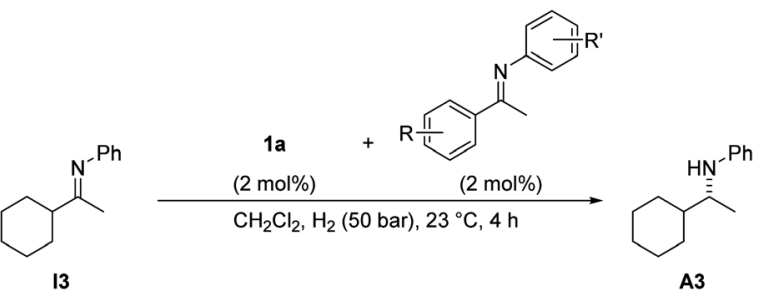

\begin{tabular}{|c|c|c|c|c|c|}
\hline Entry & Additive & $\mathrm{R}$ & $\mathrm{R}^{\prime}$ & Conv. $^{a}(\%)$ & $\mathrm{ee}^{b}(\%)$ \\
\hline 1 & I1 & $\mathrm{H}$ & $\mathrm{H}$ & $>99$ & $71(R)$ \\
\hline 2 & I4 & 2-Me & $\mathrm{H}$ & $>99$ & $69(R)$ \\
\hline 3 & I5 & $2-\mathrm{F}$ & $\mathrm{H}$ & $90-95$ & $71(R)$ \\
\hline 4 & I6 & $3-\mathrm{NO}_{2}$ & $\mathrm{H}$ & 75-99 & $48(R)$ \\
\hline 5 & I7 & $3,5-\left(\mathrm{NO}_{2}\right)_{2}$ & $\mathrm{H}$ & 10 & $15(R)$ \\
\hline 6 & I8 & $3,5-\mathrm{Me}_{2}$ & $\mathrm{H}$ & $>99$ & $3(S)$ \\
\hline 7 & I9 & $3,5^{-i} \operatorname{Pr}_{2}$ & $\mathrm{H}$ & 34 & $42(S)$ \\
\hline 8 & I10 & $3,5^{-t} \mathrm{Bu}_{2}$ & $\mathrm{H}$ & 11 & $10(R)$ \\
\hline 9 & I2 & 4-MeO & $\mathrm{H}$ & $>99$ & $67(R)$ \\
\hline 10 & I11 & $4-{ }^{t} \mathrm{Bu}$ & $\mathrm{H}$ & $>99$ & $64(R)$ \\
\hline 11 & I12 & 4-Me & $\mathrm{H}$ & $>99$ & $63(R)$ \\
\hline 12 & I13 & 4-Cl & $\mathrm{H}$ & $>99$ & $56(R)$ \\
\hline 13 & I14 & $4-\mathrm{F}$ & $\mathrm{H}$ & $>99$ & $66(R)$ \\
\hline 14 & I15 & $4-\mathrm{CF}_{3}$ & $\mathrm{H}$ & 95 & $50(R)$ \\
\hline 15 & I16 & $4-\mathrm{NO}_{2}$ & $\mathrm{H}$ & 40 & $45(R)$ \\
\hline 16 & I17 & 4-Me & $2-\mathrm{Br}$ & $>99$ & $68(R)$ \\
\hline 17 & I18 & $\mathrm{H}$ & 2-Me & $>99$ & $78(R)$ \\
\hline 18 & I19 & $\mathrm{H}$ & 2-MeO & $>99$ & $71(R)$ \\
\hline 19 & $\mathbf{I} 20$ & $\mathrm{H}$ & 3-MeO & $>99$ & $68(R)$ \\
\hline 20 & I21 & $\mathrm{H}$ & 4-MeO & $>99$ & $69(R)$ \\
\hline 21 & $\mathbf{I} 22$ & $\mathrm{H}$ & $4-\mathrm{CF}_{3}$ & $>99$ & $59(R)$ \\
\hline 22 & $\mathbf{I} 23$ & $\mathrm{H}$ & $2-{ }^{i} \mathrm{Pr}$ & $>99$ & $81(R)$ \\
\hline 23 & $\mathbf{I} 24$ & $\mathrm{H}$ & $2,6-\mathrm{Me}_{2}$ & $>99$ & $85(R)$ \\
\hline
\end{tabular}

${ }^{a}$ Determined by GC analysis. ${ }^{b}$ Determined by HPLC analysis on a chiral stationary phase.

enantioselectivity (entries 17, 22 and 23). Other iridium complexes were screened as well, but none of them reached the enantioselectivities achieved with PHOX complex 1a. ${ }^{13}$

With an optimized catalyst system in hand we studied the scope for the hydrogenation of aliphatic ketimines (Table 5). Higher conversions and enantioselectivities were obtained in all cases compared to reactions using complex 1a alone. The high reactivity of this catalyst system allowed lowering the reaction temperature to $-5{ }^{\circ} \mathbf{C}$. Under these conditions imine $\mathbf{I} 3$ furnished the product $\mathbf{A 3}$ with an improved ee of $92 \%$ and full conversion (entry 4). Isopropyl methyl ketimine $\mathbf{I 2 5}$ gave $84 \%$ ee but only $33 \%$ conversion (entry 5 ). The ee was further improved when the more bulky complex $\mathbf{1 b}$ was used as precatalyst for isobutyl methyl ketimine $\mathbf{I 2 6}$ and benzyl methyl ketimine $\mathbf{I 2 7}$ (entries 8 and 10). However, for $\alpha$-branched alkyl methyl ketimines such as I3, I25, I31 and I32, no reaction was observed with complex 1b. The sterically less demanding $n$-alkyl methyl ketimines I28, I29 and I30 gave markedly lower enantioselectivity (entries 11-13). Chemoselective hydrogenation of an imine double bond in the presence of a trisubstituted olefin can be achieved as shown in $\mathbf{I 2 9}$ (entry 12).
Table 5 Asymmetric hydrogenation of $N$-phenyl aliphatic imines

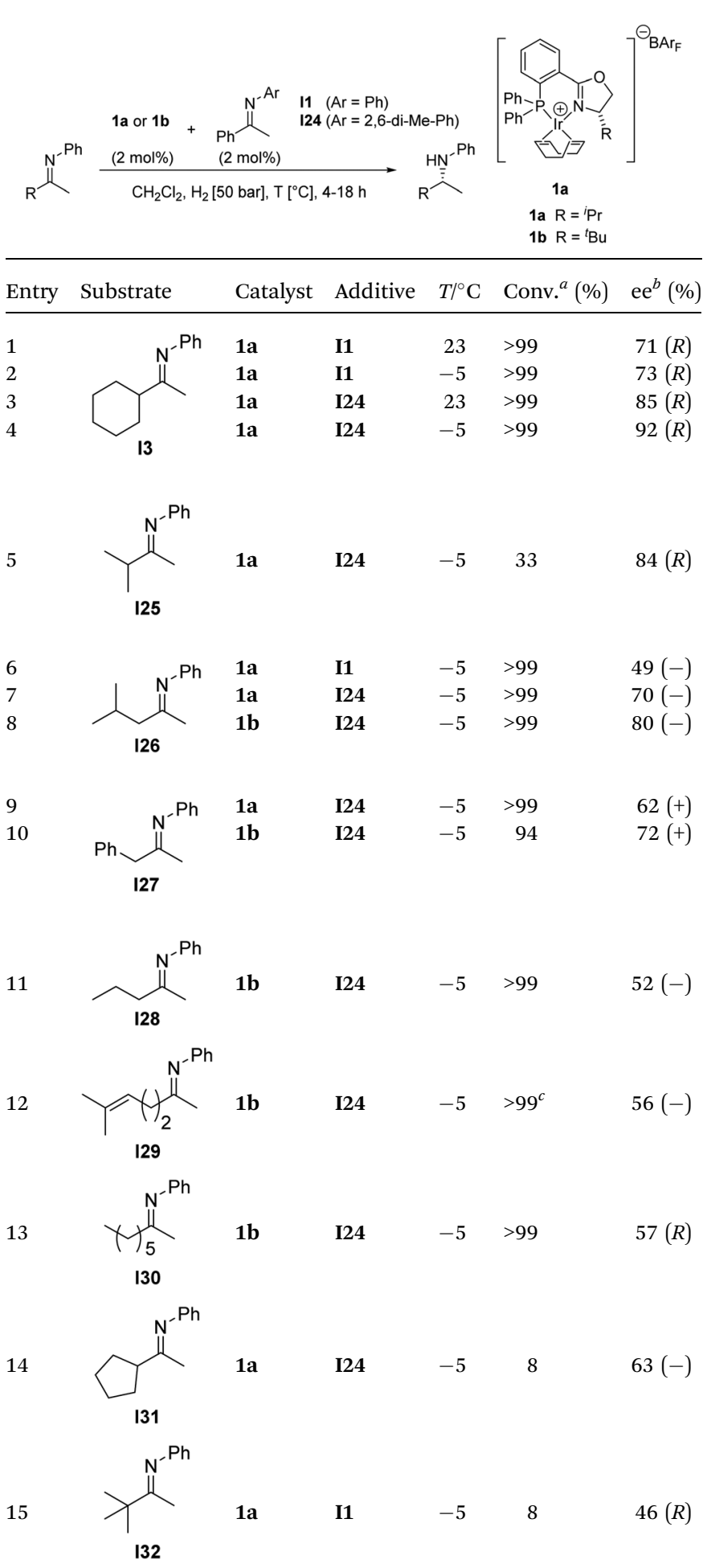

${ }^{a}$ Determined by GC analysis. ${ }^{b}$ Determined by HPLC analysis on a chiral stationary phase after purification by flash chromatography. ${ }^{c}$ Contained $<3 \%$ of fully saturated product.

To see whether the $N$-phenyl group was essential for achieving high enantioselectivity, we investigated $N$-alkyl imines I33-I36 as substrates (Table 6). Asymmetric hydrogenation of imines of this 
type has not been reported yet apart from I33. As these substrates proved to be less reactive, hydrogenations were conducted at room temperature. Using imine $\mathbf{I} 1$ as additive the $N$-benzylimine I33 furnished a moderate ee of 44\% (entry 1). An even lower enantioselectivity is observed for the $N$ - $n$-butylimine $\mathbf{I 3 4}$ (entry 2). On the other hand almost the same ee as for corresponding $\mathrm{N}$-phenylimine $\mathbf{I} 3$ was observed in the hydrogenation of $\mathbf{I 3 5}$ (entry $3)$. The $N$-cyclohexyl analogue $\mathbf{I 3 4}$ reacted with even higher enantioselectivity of $77 \%$ ee (entry 4 ), demonstrating that purely alkyl-substituted imines are suitable substrates for this catalyst system. The more bulky complex $\mathbf{1 b}$ and the sterically demanding $N$-(2,6-dimethylphenyl)imine $\mathbf{I 2 4}$ afforded lower yields and enantioselectivities with these substrates.

\section{Conclusions}

We have found that the active catalyst in the hydrogenation of acetophenone-derived imines with Ir-PHOX precatalysts is an iridacycle generated under hydrogenation conditions by cyclometalation of the substrate. Cyclometalated complexes of this type, formed in situ by addition of an equimolar equivalent of acetophenone imine, show higher reactivity and better enantioselectivity in the hydrogenation of $\mathrm{N}$-phenyl and $\mathrm{N}$-alkyl aliphatic ketimines than the corresponding Ir-PHOX complex alone. Obviously, the reaction proceeds through a pathway that differs from the catalytic cycles proposed in the literature. ${ }^{7}$ Although at present the scope is still limited, our findings indicate many opportunities for further improvement of this catalyst system by structural variation of both the chiral P,N ligand and the cyclometalated imine.

\section{Experimental section}

\section{Screening}

Imine $(0.1 \mathrm{mmol})$, catalyst $(2 \mu \mathrm{mol})$, additive $(2 \mu \mathrm{mol})$, and a stir bar were added to an oven-dried glass vial that had been placed

Table 6 Asymmetric hydrogenation of $N$-alkyl aliphatic imines

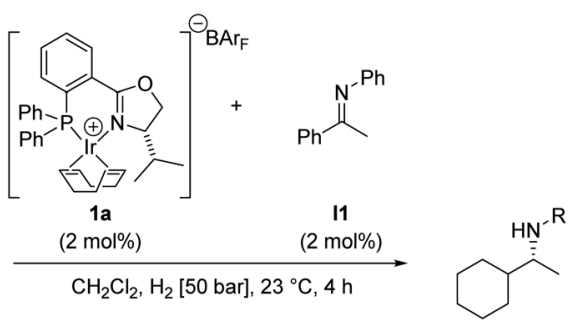

\begin{tabular}{llll}
\hline Entry & Substrate (R) & Conv. $^{a}(\%)$ & ee $^{b}(\%)$ \\
\hline 1 & $\mathbf{I 3 3}\left(\mathrm{CH}_{2} \mathrm{Ph}\right)$ & $>99$ & $44(R)^{c}$ \\
2 & $\mathbf{I 3 4}\left({ }^{n} \mathrm{Bu}\right)$ & $>99$ & $33(R)$ \\
3 & $\mathbf{I 3 5}\left({ }^{i} \mathrm{Pr}\right)$ & $>99$ & $73(R)^{d}$ \\
4 & $\mathbf{I 3 6}\left(c-\mathrm{C}_{6} \mathrm{H}_{11}\right)$ & $>99$ & $77(R)^{c}$
\end{tabular}

${ }^{a}$ Determined by GC analysis. ${ }^{b}$ Determined by GC or HPLC analysis on a chiral stationary phase after derivatisation. ${ }^{c}$ Determined after derivatisation to the 1-naphthoyl amide. ${ }^{d}$ Determined after derivatisation to the acetamide. in an autoclave $(60 \mathrm{~mL})$ and purged with argon for $5 \mathrm{~min}$. Anhydrous $\mathrm{CH}_{2} \mathrm{Cl}_{2}(1 \mathrm{~mL})$ was added by syringe under a stream of argon and the autoclave was closed. For reactions at low temperature the autoclave was immersed in a cooling bath for $60 \mathrm{~min}$ before starting the reaction. The autoclave was pressurized with hydrogen gas, hydrogen was released and the autoclave pressurized again. It was then placed on a stirring plate for the time indicated. After releasing the pressure, the solvent was evaporated under a stream of nitrogen. The residue was suspended in pentane-diethyl ether $(5: 1)$ and filtered through a short elution plug (cotton bottom, $40 \times 5 \mathrm{~mm}$ silica gel). The crude filtrate was analysed by GC for conversion before being purified by flash chromatography $\left(\mathrm{SiO}_{2}\right.$, pentane-diethyl ether (20:1), $15 \times 2 \mathrm{~cm})$ and analysed by HPLC on a chiral stationary phase for determination of the enantiomeric excess.

\section{Preparative reaction}

Imine $\mathbf{I 3}$ (1.005 g, $5 \mathrm{mmol})$, 1a (0.1 mmol), $\mathbf{I 2 4}(0.1 \mathrm{mmol})$, and a stir bar were added to a $25 \mathrm{~mL}$ Pyrex oven-dried glass vial that had been placed in an autoclave $(60 \mathrm{~mL})$ and purged with argon for $5 \mathrm{~min}$. Anhydrous $\mathrm{CH}_{2} \mathrm{Cl}_{2}(5 \mathrm{~mL})$ was added by syringe under a stream of argon and the autoclave was closed. The autoclave was immersed in a cooling bath for $60 \mathrm{~min}$ at $-5{ }^{\circ} \mathrm{C}$ before it was pressurized with hydrogen gas. Hydrogen was released and the autoclave pressurized again before being placed on a stirring plate for $18 \mathrm{~h}$. After pressure release the reaction mixture was transferred to a $50 \mathrm{~mL}$ round-bottom flask and solvents removed under reduced pressure. The residue was suspended in pentane-diethyl ether $(20: 1)$ and purified by flash chromatography $\left(\mathrm{SiO}_{2}\right.$, pentane-diethyl ether (10:1), $\left.21 \times 3 \mathrm{~cm}\right)$. Solvents were removed under reduced pressure and the residue was dried in vacuo to afford A3 (998 mg, $4.92 \mathrm{mmol}, 98 \%$ ).

\section{Acknowledgements}

Support of this work by the Swiss National Science Foundation $(S N F)$ and the Federal Commission for Technology and Innovation $(K T I)$ is gratefully acknowledged. We thank Dr Markus Neuburger for the crystal structure analysis, Robin Wehlauch for synthetic contributions and Prof. Dr Klaus Dittrich from $B A S F$ for generous gifts of chemicals.

\section{Notes and references}

1 J.-H. Xie, S.-F. Zhu and Q.-L. Zhou, Chem. Rev., 2011, 111, 1713; for early work on asymmetric imine hydrogenation, see: C. A. Willoughby and S. L. Buchwald, J. Am. Chem. Soc., 1992, 114, 7562; C. A. Willoughby and S. L. Buchwald, J. Am. Chem. Soc., 1994, 116, 8952; C. A. Willoughby and S. L. Buchwald, J. Am. Chem. Soc., 1994, 116, 11703; Y. N. C. Chan and J. A. Osborn, J. Am. Chem. Soc., 1990, 112, 9400; F. Spindler, B. Pugin and H.-U. Blaser, Angew. Chem., Int. Ed. Engl., 1990, 29, 558; J. Bakos, I. Toth, B. Heil, G. Szalontai, L. Parkanyi and V. Fulup, J. Organomet. Chem., 1989, 370, 263; for more recent work, see A. Trifonova, J. S. Diesen and P. G. Andersson, Chem.-Eur. J., 2006, 12, 2318-2328; C. Moessner and C. Bolm, Angew. Chem., Int. Ed., 
2005, 44, 7564; T. Imamoto, N. Iwadate and K. Yoshida, Org. Lett., 2006, 8, 2289; S.-F. Zhu, J.-B. Xie, Y.-Z. Zhang, S. Li and Q.-L. Zhou, J. Am. Chem. Soc., 2006, 128, 12886; N. Mrsic, A. J. Minnaard, B. L. Feringa and J. G. de Vries, J. Am. Chem. Soc., 2009, 131, 8358; Z. Han, Z. Wang, X. Zhang and K. Ding, Angew. Chem., Int. Ed., 2009, 48, 5345.

2 H.-U. Blaser, Adv. Synth. Catal., 2002, 344, 17.

3 S. Zhou, S. Fleischer, K. Junge and M. Beller, Angew. Chem., Int. Ed., 2011, 50, 5120.

4 C. Q. Li, C. Wang, B. Villa-Marcos and J. L. Xiao, J. Am. Chem. Soc., 2008, 130, 14450.

5 I. Storer, D. E. Carrera, Y. Ni and D. W. C. MacMillan, J. Am. Chem. Soc., 2006, 128, 84; M. Reuping, E. Sugiono, C. Azap, T. Theissmann and M. Bolte, Org. Lett., 2005, 7, 378; S. Hoffman, A. M. Seayad and B. List, Angew. Chem., Int. Ed., 2005, 44, 7424; K. Saito and T. Akiyama, Chem. Commun., 2012, 48, 4573.

6 (a) P. Schnider, G. Koch, R. Prétôt, G. Wang, F. M. Bohnen, C. Krüger and A. Pfaltz, Chem.-Eur. J., 1997, 3, 887; (b) A. Baeza and A. Pfaltz, Chem.-Eur. J., 2010, 16, 4003.
7 K. H. Hopmann and A. Bayer, Organometallics, 2011, 30, 2483.

8 C. Mazet, S. P. Smidt, M. Meuwly and A. Pfaltz, J. Am. Chem. Soc., 2004, 126, 14176.

9 Crystal structure: see ESI. $\uparrow$

10 The collected data was of insufficient quality to allow accurate structure determination; see ESI. $\dagger$

11 (a) P. Marcazzan, B. O. Patrick and B. R. James, Russ. Chem. Bull., 2003, 52, 2715. See also (b) J. F. van Baar, K. Vrieze and D. J. Stufkens, J. Organomet. Chem., 1975, 85, 249; (c) M. Martín, E. Sola, S. Tejero, J. L. Andrés and L. A. Oro, Chem.-Eur. J., 2006, 12, 4043.

12 P. Marcazzan and B. R. James, React. Kinet. Catal. Lett., 2009, 98, 193.

13 See ESI. $†$

14 Control experiments showed that even 0.1 mol\% of $\mathbf{~ I 1}$ or $\mathbf{A 1}$ would have been detected by GC analysis.

15 Cyclometalation of $\mathbf{I 1 0}$ was not observed in a preparative reaction, which could explain the low conversion and ee in this case. 